\title{
Actinomycosis, a lurking threat: a report of 11 cases and literature review
}

\author{
Catarina Oliveira Paulo ${ }^{[1]}$, Sofia Jordão ${ }^{[1]}$, João Correia-Pinto ${ }^{[2],}$ \\ Fernando Ferreira ${ }^{[3]}$ and Isabel Neves ${ }^{[1]}$
}

\begin{abstract}
[1]. Infectious Diseases Unit, Medical Department, Hospital Pedro Hispano - Matosinhos Local Health Unit, Matosinhos, Portugal. [2]. Department of Anatomical Pathology, Hospital Pedro Hispano - Matosinhos Local Health Unit, Matosinhos, Portugal.

[3]. Department of General Surgery, Hospital Pedro Hispano - Matosinhos Local Health Unit, Matosinhos, Portugal
\end{abstract}

\begin{abstract}
Actinomycosis remains characteristically uncommon, but is still an important cause of morbidity. Its clinical presentation is usually indolent and chronic as slow growing masses that can evolve into fistulae, and for that reason are frequently underdiagnosed. Actinomyces spp is often disregarded clinically and is classified as a colonizing microorganism. In this review of literature, we concomitantly present 11 cases of actinomycosis with different localizations, diagnosed at a tertiary hospital between 2009 and 2016. We outline the findings of at least one factor of immunosuppression in $>90 \%$ of the reported cases.
\end{abstract}

Keywords: Actinomycosis. Immunosuppression. Infection. Underdiagnosis.

\section{INTRODUCTION}

Actinomycosis is uncommon, indolent, and chronic, and is caused by the microorganism Actinomyces spp. Its incidence has diminished globally due to improved oral hygiene and the development of antibiotics. Actinomycosis is frequently underdiagnosed because of its clinical and imagiological mimicry of other infectious diseases (e.g. nocardiosis, tuberculosis) and malignancies ${ }^{1,2}$.

Due to its filamentous morphology, this gram-positive anaerobe was previously classified as a fungus. Presently it is considered as a member of the order Actinomycetales (grouped with nocardias, streptomyces, and micobacterias), and is a part of the oral, gastrointestinal and urogenital commensal flora $^{3-6,}$. Actinomyces is known for its ability to evolve into invasive masses with or without fistulous tracts when there are minor breaches in tissue integrity (e.g. trauma, radiotherapy, inflammatory perforation $)^{7,8}$.

The most common pathogenic species is Actinomyces israelii. Occasionally Actinomyces naeslundii, Actinomyces odontolyticus, Actinomyces viscosus, Actinomyces meyeri (most frequent agent of disseminated disease), and rarely Actinomyces neuii (these last two species are typically non filamentous $)^{9,10}$ among others ${ }^{11}$, have been found as the etiological agents.

Corresponding author: Dra. Catarina Oliveira Paulo.

e-mail: catarinaoliveira.paulo@ulsm.min-saude.pt

Received 20 August 2017

Accepted 28 December 2017
There are multiple possible focalizations, the most frequently described being oro-cervicofacial (55\%) and abdominalpelvic (20\%). The abdomino-pelvic focalization is frequently associated with a past history of perforated appendicitis ${ }^{12,13}$ or with the prolonged use of an intrauterine device (IUD) $)^{14,15}$.

In $15 \%$ of cases, the location is thoracic, including intrathoracic organs and the thoracic wall ${ }^{16}$. Cases of musculoskeletal actinomycosis, such as that of the long bones are rare, and are typically described as chronic sclerotic or spherical osteomyelitis ${ }^{17,18}$. Such infections originate from infected adjacent tissues or, less frequently, following hematogenous dissemination. Infections of the urogenital tract ${ }^{19}$, eye ${ }^{20-22}$, skin, and disseminated disease ${ }^{23,24}$ focalized in the central nervous system $(\mathrm{CNS})^{25}$ are rare; a case of chronic lymphadenitis has also been reported ${ }^{26}$. Only vertical transmission has been reported, and two cases of neonatal sepsis have been described: one caused by A. neuii and the other (species unidentified) with uterine cerclage as the identifiable risk factor ${ }^{27,28}$.

There seems to be a relationship between development of infection and an immunosuppression risk factor underlining it, as it has been described in patients with human immunodeficiency virus (HIV) infection, leukemia, solid organ malignancies, and others ${ }^{29-33}$. Based on the localization of the infection, its extension, time of evolution, and basal status of the patient, the disease may manifest in multiple forms, and may present with fever, asthenia, and progressive loss of sense of well-being.

The biochemical profile is non-specific and varies with the clinical manifestation of the disease, though anemia, elevated reactive $\mathrm{C}$ protein $(\mathrm{RCP})$ and/or changes in the sedimentation rate (SR), and mild leucocytosis are common. Radiological 
findings might include presence of masses, cold abscesses, or fistulae, depending on the time-frame and disease progression.

Diagnosis is based on characteristic histological and/or microbiological findings (the latter helps in identifying the species and antimicrobial susceptibility).

Histology is characterized by the presence of Grampositive filamentous structures and sulfuric granules. Gomori methenamine-silver and Giemsa stains are commonly used, and a rapid direct immunofluorescence method has also been developed to identify a species-specific antibody ${ }^{25}$ Pathogen identification is successful in less than $50 \%$ of cases, owing to the recent use of antibiotics, overgrowth of other microorganisms, and inadequate diagnostic methodology. Gram staining is thus considered a more sensitive technique than is culture. Culturing Actinomyces requires a selective anaerobe agar maintained at $37^{\circ} \mathrm{C}$, and a growth time of 3 weeks. Swab techniques are reported to be ineffective for culturing this bacterium. In some European reference laboratories, the preferred method for Actinomyces spp. identification is $16 \mathrm{~s}$ ribosomal ribonucleic acid (rRNA) sequencing ${ }^{25}$.

Antibiotic treatment duration depends on infection localization and severity, and concomitant surgical excision or IUD removal (in appropriate cases) may be required.

Actinomyces spp. is known to be susceptible to penicillins and to other B-lactams, with the exception of oxacillin, dicloxacillin, and cephalexin; Actinomyces europaeus and graevenitzii are reported to be resistant to ceftriaxone, and other species with intermediate susceptibility to cephalosporins have also been described. This anaerobe is also susceptible to doxycycline, clindamycin, erythromycin and clarithromycin, linezolide, and tigecicline ${ }^{25,34,35}$. Aminoglycosides, metronidazole, and quinolones are not reported to show adequate bactericidal activity against Actinomyces spp. ${ }^{36}$. Although the organism is multisusceptible, in most cases of infection, an initial intravenous treatment with antibiotics for 2 to 6 weeks (preferably with penicillin $\mathrm{G}$ ) with subsequent prolonged oral treatment for 6 to 12 month (amoxicillin 2g/day divided in 4 doses) is required ${ }^{25,35}$.

Disease prognosis depends on the focalization (worse in CNS disease), extension of infection, the patient's baseline comorbidities, and delay in diagnosis. The current mortality rates are reported to vary from 0 to $28 \%{ }^{25}$.

We present here 11 cases of Actinomycosis diagnosed between 2009 and 2016 at a tertiary referral hospital situated North of Porto-Portugal, with different clinical presentations and subjacent co-morbidities, as summarized in Table 1.

\section{CASES}

Case 1. A 43-year-old woman, diagnosed with a locally advanced esophagic epidermoid carcinoma, was treated with radiotherapy and chemotherapy (QT). Two months after the last session, while maintaining a mass that was considered unresectable, she visited the emergency department with upper airway obstruction. Fever and other symptoms were absent. Upper gastrointestinal endoscopy (UGIE) revealed a vegetative ulcerated mass with a circumferential cervical edema. Histological examination of the biopsy revealed necrotic tissue with Actinomyces spp. colonies, with no evidence of malignancy (Figure 1). Treatment was administered for 2 weeks with intravenous penicillin, resulting in resolution of the obstruction and the patient continued the treatment for 12 more months with oral amoxicillin (500mg every 6 hours), with no recurrence.

Case 2. A 34-year-old man with a history of chronic alcohol ingestion was hospitalized with sepsis after a gastric ulcer perforation, and presented with secondary peritonitis. Pyroloplasty and vagotomy were performed, and he was treated empirically with piperacillin/tazobactam for 7 days. Abdominal pain and fever were present, despite a favorable initial recovery. A second exploratory laparotomy was performed, and abscesses in the intestinal loops as well as dispersed intra-abdominal pus were observed, with no evidence of dehiscence of the past surgical procedure. Actinomyces naeslundii was identified in the peritoneal fluid, and he was treated with intravenous ampicillin (800mg every 8 hours) for 3 weeks (he had received a 10-day course of empirical carbapenem previously), resulting in analytical and clinical resolution. Oral amoxicillin treatment at $2 \mathrm{~g}$ /day was administered for 6 months with no clinical intercurrences.

Case 3. A 37-year-old obese man with a clinical history of perforated appendicitis developed an incisional hernia shortly after presenting with secondary peritonitis in 2002 . The hernia was surgically approached multiple times, and multiple unsuccessful attempts to insert a mesh were made until 2013. Recurring entero-cutaneous fistulae were detected.

In 2010, after another attempt at curing the fistulous tract, a segmental enterectomy was performed, resulting in yet another recurrence of a fistula in 2011. The fistula was observed to extend through the abdominal wall tissue $(12 \times 9 \mathrm{~mm})$ and progress to the adjacent cecum wall.

A final intricate surgical intervention in 2013 was performed for the correction of a complex hernia, with extensive resection of fibrous adhesions around the abdominal viscera. The old mesh was extracted and sent for microbiological analysis (negative result) and histological examination. Microscopically, Actinomyces spp colonies were identified, and he completed 3 weeks of intravenous penicillin followed by amoxicillin at $2 \mathrm{~g} /$ day for 6 months, with no recurrence of fistulous tracts or herniation of the abdominal wall.

Case 4. An 81-year-old woman with a clinical history of an oophorectomy 20 years earlier and no other co-morbidities, presented with an intestinal obstruction and constant pain (one week duration) in the right lower quadrant. No other symptoms were present. In an abdominal computed tomography (CT) scan a heterogeneous mass $(4 \times 5 \mathrm{~cm})$ involving the appendix, terminal ileum, and cecum (Figure 2) was observed and a locally advanced malignancy was suspected. Laparotomy confirmed the mass, and an associated perforation of the intestinal tract was suspected, leading to a right hemicolectomy. Histology of the surgical specimen revealed a peri-apendicular mass with Actinomyces spp. colonies and no tissue perforation (appendicular or intestinal) or malignancy. She was given 
TABLE 1: Summary of clinical and diagnostic characteristics and treatment of the cases described.

\begin{tabular}{|c|c|c|c|c|c|c|}
\hline Cases & $\begin{array}{l}\text { Age } \\
\text { (years) }\end{array}$ & Focalization & Diagnosis & $\begin{array}{l}\text { Immunosuppressive } \\
\text { factors }\end{array}$ & $\begin{array}{l}\text { Intravenous } \\
\text { treatment }\end{array}$ & $\begin{array}{l}\text { Oral treatment with } \\
\text { amoxicillin ( } 2 \mathrm{~g} / \text { day) }\end{array}$ \\
\hline 1 & 43 & Esophageal & Histology & Epidermoid carcinoma & 2 weeks of penicillin $\mathrm{G}$ & 12 months \\
\hline 2 & 34 & Abdominal & $\begin{array}{l}\text { Peritoneal fluid Actinomyces } \\
\text { naeslundii } \\
\text { (anaerobe media Brucella } \\
\text { agar at } 37^{\circ} \mathrm{C} \text {; identified } \\
\text { VITEK® 2) }\end{array}$ & Alcoholism & 3 weeks ampicillin & $\begin{array}{l}6 \text { months and } \\
\text { abandonment of } \\
\text { consultation }\end{array}$ \\
\hline 3 & 37 & Abdominal wall & Histology & Morbid Obesity & $\begin{array}{l}3 \text { weeks of penicillin } \\
G\end{array}$ & 6 months \\
\hline 4 & 81 & Abdominal & Histology & $>65$ years & Multiple & 12 months \\
\hline 5 & 81 & Neck of femur & Histology & > 65 years, obesity & 3 weeks ampicillin & 12 months \\
\hline 6 & 67 & Tongue & Histology & HIV infection & - & 6 months \\
\hline 7 & 62 & Gastric & Histology & $\begin{array}{l}\text { High-grade gastric non- } \\
\text { Hodgkin lymphoma; } \\
\text { Alcoholism }\end{array}$ & 4 weeks ampicillin & 12 months \\
\hline \multirow[t]{2}{*}{8} & 38 & Abdominal & Intra-abdominal abscess & & $\begin{array}{l}3 \text { weeks ertapenem + } \\
4 \text { weeks ampicillin }\end{array}$ & 12 months \\
\hline & & & $\begin{array}{c}\text { Actinomyces naeslundii } \\
\text { (anaerobe media Brucella } \\
\text { agar and aerobe media } \\
\text { blood agar at } 37^{\circ} \mathrm{C} \text {; identified } \\
\text { VITEK® 2) }\end{array}$ & Crohn's disease & & \\
\hline 9 & 49 & Abdominal & Histology & Unknown & 4 weeks ampicillin & 12 months \\
\hline 10 & 63 & $\begin{array}{l}\text { Synovial cyst of the } \\
\text { ischial tuberosity }\end{array}$ & $\begin{array}{l}\text { Blood culture Actinomyces } \\
\text { naeslundii }\end{array}$ & Corticotherapy & 4 weeks ampicillin & 6 months \\
\hline 11 & 26 & Pulmonary & Pus & Type 1 diabetes mellitus & 4 weeks ampicillin & 8 months \\
\hline & & & Actinomyces spp & & & \\
\hline
\end{tabular}

HIV: human immunodeficiency virus.

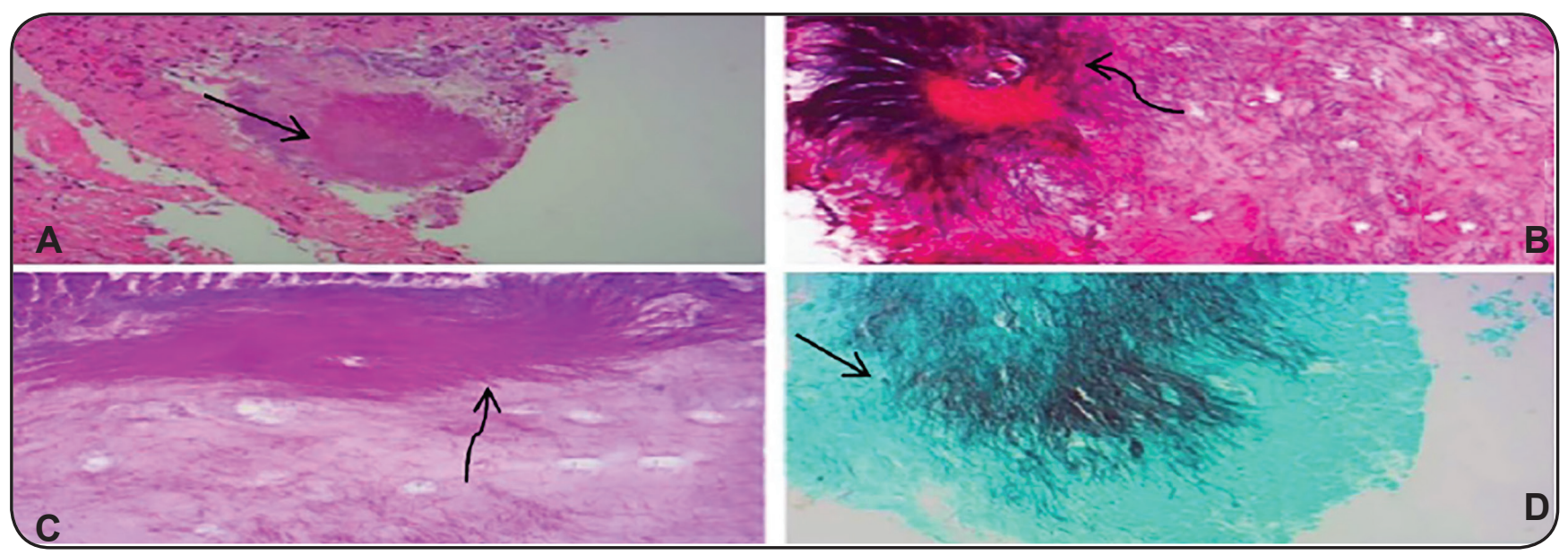

FIGURE 1: Characteristic sulfur granule of actinomycosis (A: H\&E; 200X). Histochemical stains reveal that these granules are composed of delicate, branched, filamentous bacteria (B: Gram Stain; C: PAS stain; D: Grocott stain; 200X). H\&E: hematoxylin and eosin; PAS: periodic acid-Schiff. 
doxycycline for suspected inflammatory pelvic disease. The patient improved clinically and was discharged.

After one month she presented with fever and an intestinal pseudobstruction; a new inflammatory mass $(4 \times 4 \mathrm{~cm})$ was observed; an anastomotic dehiscence was excluded and she was treated with piperacillin/tazobactam with clinical improvement. She was then discharged and prescribed amoxicillin/clavulanate treatment in sub-therapeutic doses. After two months, an intermittent febrile syndrome with no obvious focus was apparent, and she once again returned to the emergency department. Elevated RCP and SR values with no radiological changes were observed. After a consultation with the Infectious Diseases unit and exclusion of other diagnoses (namely tuberculosis in a previous biopsy), amoxicillin was initiated at $2 \mathrm{~g} /$ day, and she completed 12 months of treatment with clinical resolution.

Case 5. An 81-year-old obese woman with no other comorbidities was admitted for a pathological fracture of the femoral neck with no other symptoms. Necrosis of the femoral head was observed and histology revealed lesions suggesting acute necrotizing osteomyelitis with Actinomyces spp. (Figure 3). She was treated for 3 weeks with intravenous ampicillin followed by amoxicillin at $2 \mathrm{~g}$ /day for 12 months. The infection was resolved and partial mobility was restored.

Case 6. A 67-year-old man with a history of stomatitis and tooth decay was examined at an otolaryngology consultation for a relapsing ulcerovegetative lesion of the tongue. A contrast enhancement cervical CT showed an invasive lesion $(2 \mathrm{~cm})$. Biopsy of the tissue revealed tissue necrosis and abundance of Actinomyces spp colonies, with no evidence of malignancy. Due to the rare diagnosis, he was screened for and diagnosed with an HIV infection. He was treated for 6 months with oral amoxicillin (2g/day) and associated antiretroviral therapy, with total resolution of the described lesion.

Case 7. A 62-year-old man with high alcohol and tobacco consumption and with no other clinical relevant history was hospitalized due to a stroke secondary to anemia. A diagnostic UGIE investigation revealed a gastric ulcerated mass in the greater curvature, highly suggestive of a malignancy (Figure 4). A gastrectomy was scheduled, but postponed when multiple gastric and duodenal tissue biopsies showed the presence of Actinomyces spp and no evidence of a malignant tumor. He completed 4 months of ampicillin at $1,250 \mathrm{mg} / 6$ hours followed by oral amoxicillin treatment at $2 \mathrm{~g} /$ day. After 3 months of consolidation therapy a new UGIE was performed and showed the same described mass. Histological examination showed no malignancy, and the patient described mild abdominal pain in the left iliac fossa, though no anemia or relapse of fever was observed. An abdominal CT revealed an augmentation of the described mass $(16 \times 10 \mathrm{~cm})$ with extensive central necrosis along the lesser gastric curvature and antrum and invasion into the adjacent tissues. Owing to the gastric outlet obstruction and possible resistant actinomycosis (no microbiological identification was successful with previous biopsies) or malignancy, he underwent an elective subtotal gastrectomy. Macroscopically there was ascites, macronodular cirrhosis of

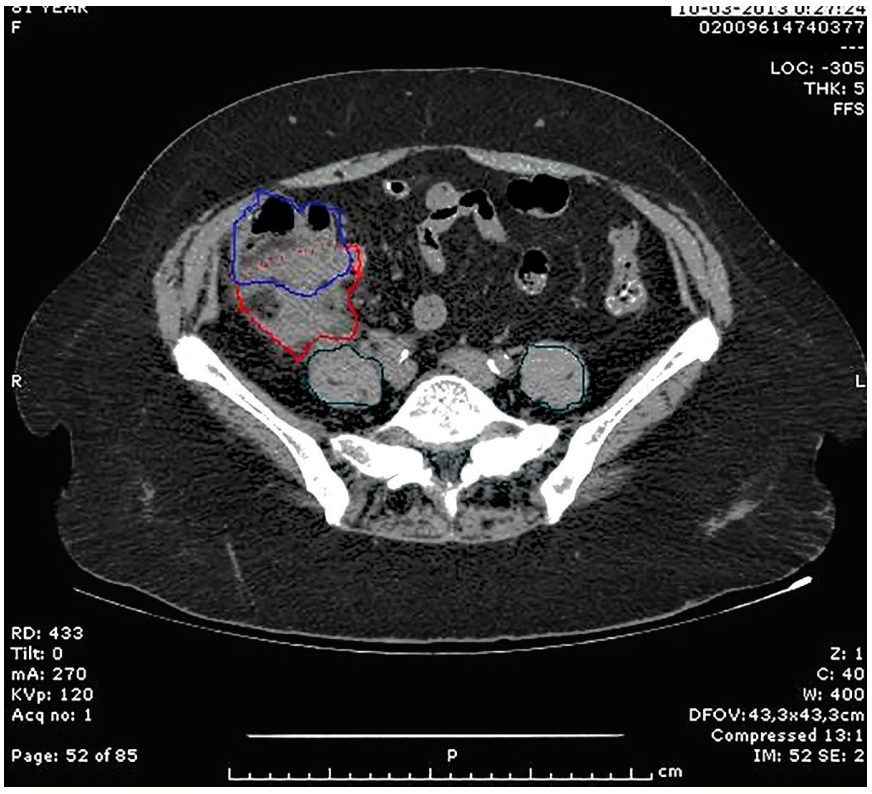

FIGURE 2: A computed tomography (CT) scan with an intra-abdominal mass.

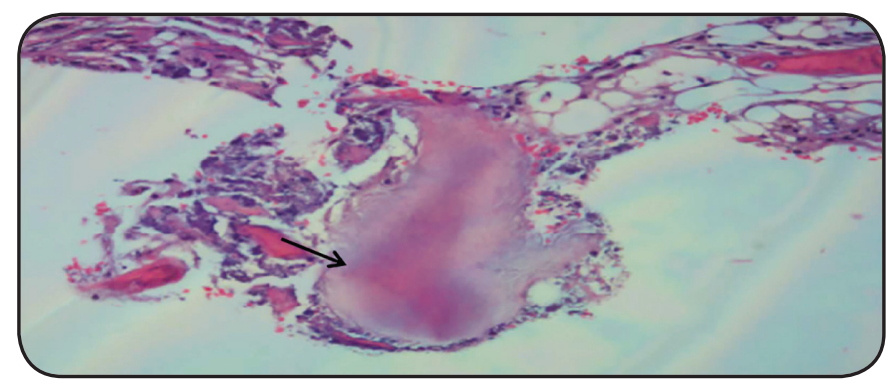

FIGURE 3: Bony trabeculae with presence of Actinomyces spp. colony (H\&E; 400X). H\&E: hematoxylin and eosin.

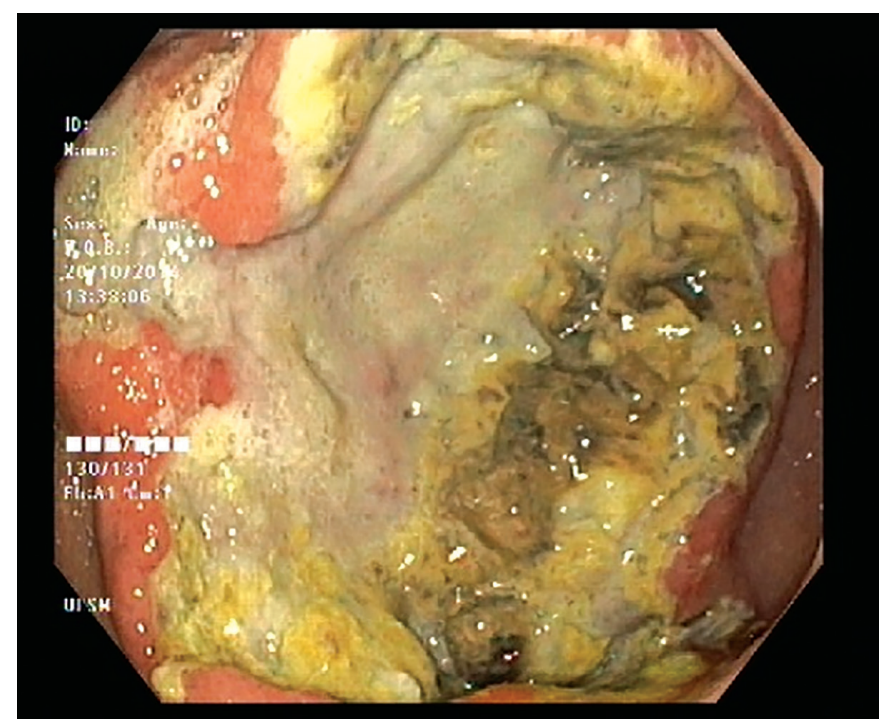

FIGURE 4: Ulcerated mass in the gastric body in the $1^{\text {st }}$ upper gastrointestinal endoscopy (UGIE). 
the left hepatic lobe with collateral circulation adjacent to the mass. The mass showed adherence to the gallbladder and the transverse mesocolon, with no peritoneal implants. Histological and immunohistochemical findings suggested high-grade gastric non-Hodgkin lymphoma and presence of colonies of Actinomyces spp.

He completed 6 cycles of chemotherapy and 12 months of antimicrobial therapy with good clinical response, involution of the gastric mass, and is in remission.

Case 8. A 38-year-old man with a history of Crohn's disease treated with mesalazine, was electively submitted to a right hemicolectomy and segmental enterectomy for recurring episodes of intestinal pseudobstruction. He presented with additional complications of an intra-abdominal abscess and presence of Enterococcus faecium (susceptible to linezolide) and Actinomyces naeslundii. At the time of the diagnosis, he had completed 3 weeks of empirical treatment with ertapenem with clinical improvement, and was discharged with prolonged oral amoxicillin treatment at $2 \mathrm{~g} / \mathrm{day}$ and 2 weeks of linezolide at $600 \mathrm{mg}$ every 12 hours. At second week of treatment, purulent discharge through the surgical drain was observed and he was promptly hospitalized to complete a 4 week intravenous treatment with ampicillin at $1 \mathrm{~g}$ every 8 hours with cessation of drainage and removal of the drain. He then completed 12 months of oral antibiotic with complete clinical resolution.

Case 9. A 49-year-old woman fitted with an IUD for more than 10 years, with no other relevant clinical history was observed at the emergency department for a month-old growing mass in the left iliac fossa, now with associated pain and no other symptoms. A magnetic resonance image showed a vascularized mass of non-well defined borders $(6 \times 8 \mathrm{~cm})$ that invaded the rectus abdominis and external oblique muscles, with an apparent continuity with the sigmoid colon that confirmed findings of a previous CT scan. Since a malignant neoplasm was suspected, an exploratory laparotomy was performed and a mass with invasion of the left ovary, fallopian tube, and the bladder cupula was observed, as well as multiple soft adjacent adenopathies. An enterectomy, left colectomy with bilateral oophorectomy, and a cystorrhaphy were performed. All surgical specimens revealed no malignant lesions and showed the presence of Actinomyces spp colonies.

She completed 4 weeks of intravenous ampicillin at $750 \mathrm{mg}$ every 6 hours with clinical resolution. The IUD was removed and she continued therapy for 12 months with amoxicillin at $2 \mathrm{~g} /$ day orally with no recurring masses.

Case 10. A 63-year-old woman, medicated with corticosteroids for a rheumatic polymyalgia, presented with a perianal fistula along with a five-month-old adjacent growing mass in the left gluteus muscle (approximately $9 \mathrm{~cm}$ ). When a fistulectomy was completed, it excluded a fistulous tract and the mass was interpreted as a synovial cyst of the ischial tuberosity with radiological signs of chronic bursitis. She was discharged and returned after one month for a dehiscence of the surgical wound with slow healing, continuous discharge, and occasional fever. She was empirically treated with multiple cycles of antibiotic ( $\beta$-lactams) therapy in the short-term with no sustained clinical improvement. Actinomyces naeslundii was then identified in a blood culture, but was initially considered as colonization or contamination of the sample. After 3 months, a new surgical procedure was needed and the Infectious Diseases Unit was consulted. She started intravenous treatment for musculoskeletal actinomycosis with clinical and analytical resolution and complete healing of the initial surgical wound infection.

Case 11. A 26-year-old woman with poorly controlled type 1 diabetes mellitus and periodontal disease was hospitalized for a month with community-acquired pneumonia that manifested with right pleuritic pain and elevated inflammatory markers. No cough or sputum was observed, and she was afebrile. In a thoracic CT angiograph, two peripheral consolidations were observed in the inferior right pulmonary lobe. No microorganism could be identified, and she was empirically treated with amoxicillin/clavulanate and azithromycin for 10 days with clinical improvement.

Two months after this clinical presentation, she developed a productive cough with purulent sputum, fever, right pleuritic pain, and malaise. She was hospitalized in the intensive care unit with diabetic ketoacidosis precipitated by a respiratory infection, and was diagnosed concomitantly with a right pulmonary abscess. A diagnostic aspiration puncture was performed, resulting in a complication with bronchogenic dissemination and resultant bilateral pneumonia. Both Streptococcus anginosus and Actinomyces spp. were isolated in the pus. Adequate prolonged therapy with oral amoxicillin at $2 \mathrm{~g}$ /day was administered for eight months and resulted in clinical and radiological resolution.

In all described cases an HIV infection was excluded, with the exception of case 6. It is interesting that in all cases, the increase in inflammatory marker levels depended on the focalization of infection and underlying pathologies, and such an increase was notably mild. We would emphasize that the decrease in the SR correlated significantly with resolution of infection, even more so than did a change in CRP.

\section{CONCLUSION}

In all of the presented cases of actinomycosis it is clear that a late diagnosis of this infection is still an ongoing difficulty, delaying appropriate treatment and increasing infection duration. Underdiagnosed infections lead to increased morbidity (namely with unnecessary surgeries) because of the organism's indolent behavior, capacity for mimicking malignancies, and the common belief that Actinomyces spp detection in biological samples is due to colonization.

An intravenous treatment for at least 2 weeks is indicated for chronic disease, and as in current study cases, an excellent response to high doses of penicillins may be expected. Penicillin, which is the gold standard for treatment, was also effective in case number 8, which was refractory to ertapenem. Importantly, in most cases, histology was essential for definitive diagnosis, considering that antibiotic susceptibility and minimum inhibitory concentrations could not be established.

The probable origin of infection was identifiable in most cases and tissue disintegration/minor trauma was usually 
involved. The cause of the trauma varied, and included factors like: stomatitis and tooth decay, inflammatory bowel disease, radiotherapy, alcohol abuse, previous perforated appendicitis and oophorectomy, and presence of an IUD. In two cases of musculoskeletal manifestations the origin of infection was unclear.

The vast majority of patients presented with at least one immunosuppressive risk factor for development of disease (HIV infection, malignancies, iatrogenic immunosuppression, obesity, diabetes mellitus, chronic alcoholism, and age $>65$ years), which has also been described in the literature ${ }^{29,30-33}$. Our findings suggest that all cases of Actinomycosis may involve an underlying immunosuppressive condition, and thus we recommend that such patients be screened for HIV infection and malignancies.

A multidisciplinary approach to treat such patients and communication between medical, surgical, and laboratory teams will allow an early diagnosis and consequent adequate therapy and timely disease resolution. This approach will likely help avoid unnecessary surgeries and reduce recurrence and morbidity observed in underdiagnosed and undertreated patients.

\section{Acknowledgments}

We thank $\mathrm{Dr}^{\mathrm{a}}$ Maria João Gonçalves for her impeccable clinical management of the patients in the aforementioned case reports that were at her responsibility.

\section{Conflict of interest}

The authors declare that there is no conflict of interest.

\section{REFERENCES}

1. Sehouli J, Stupin JH, Schlieper U, Kuemmel S, Henrich W, Denkert $\mathrm{C}$, et al. Actinomycotic inflammatory disease and misdiagnosis of ovarian cancer. A case report. Anticancer Res. 2006;26(2C): 1727-31.

2. Scribner Jr DR, Baldwin J, Johnson GA. Actinomycosis mimicking a pelvic malignancy. A case report. J Reprod Med. 2000;45(6): 515-8.

3. Sarkonen N, Kononen E, Summanen P, Kanervo A, Takala A, Jousimies-Somer H. Oral colonization with Actinomyces species in infants by two years of age. J Dent Res. 2000;79(3):864-7.

4. Colebrook L. A report upon 25 cases of actinomycosis, with especial reference to vaccine therapy. Lancet. 1921;197(5096):893-99.

5. Naeslund C. Experimentelle studien $u$ ber die aetiologie und pathogenese der actinomycosis. Acta Pathol Microbiol Scand. 1931;7(suppl 6):1-156.

6. Nikolaitchouk N, Hoyles L, Falsen E, Grainger JM, Collins M. Characterization of Actinomyces isolates from samples from the human urogenital tract: description of Actinomyces urogenitalis sp. nov. Int J Syst Evol Microbiol. 2000;50(Pt 4):1649-54.

7. Postal A, Detry O, Louis E, Hardy N, Belarche J, Jacquet N. Ileocaecal actinomycosis: report of a case simulating complicated inflammatory bowel disease. Acta Gastroenterol Belg. 2001;64(4):318-20.

8. Abdalla J, Myers J, Moorman J. Actinomycotic infection of the oesophagus. J Infect. 2005;51(2):E39-43.
9. Gómez-Garcés JL, Burillo A, Gil Y, Sáez-Nieto JA. Soft tissue infections caused by Actinomyces neuii, a rare pathogen. J Clin Microbiol. 2010;48(4):1508-9.

10. von Graevenitz A. Actinomyces neuii: review of an unusual infectious agent. Infection. 2011;39(2):97-100.

11. Könönen E, Wade WG. Actinomyces and related organisms in human infections. Clin Microbiol Rev. 2015;28(2):419-42.

12. Putman HC, Dockerty MB, Waugh JM. Abdominal actinomycosis. Surgery. 1950;28:781-800.

13. Garner JP, Macdonald M, Kumar PK. Abdominal actinomycosis. Int J Surg. 2007;5(6):441-8

14. Chatwani A, Amin-Hanjani S. Incidence of actinomycosis associated with intrauterine devices. J Reprod Med. 1994; 39(8):585-7.

15. Duguid HL. Actinomycosis and IUDs. IPPF Med Bull. 1983; 17(3):1-2.

16. Karateke F, Ozyazici S, Menekşe E, Daş K, Ozdoğan M. Unusual presentations of actinomycosis; anterior abdominal wall and appendix: report of three cases. Balkan Med J. 2013;30(3):315-7.

17. Kadish LJ, Muller CJB, Mezger H. Chronic sclerosing osteomyelitis in a long bone caused by actinomycosis. A case report. S Afr Med J. 1982;62(18):658-9.

18. Rothschild B, Naples V, Barbian L. Bone manifestations of actinomycosis. Ann Diagn Pathol. 2006;10(1):24-7.

19. Lee KR, Ko YS, Yu JW, Yoon CY, Kim CH, Yoon DK. Primary vesical actinomycosis: a case diagnosed by multiple trans-abdominal needle biopsies. J Korean Med Sci. 2002;17(1):121-4.

20. Mehrotra N, Baidya A, Brijwal M, Aggarwal R, Chaudhry R. Actinomycosis of eye: forgotten but not uncommon. Anaerobe. 2015;35(Pt B):1-2.

21. Raman VS, Evans NMA, Shreshta B, Cunningham R. Chronic postoperative endophthalmitis caused by Actinomyces neuii. J Cataract Refract Surg. 2004;30(12):2641-3.

22. Garelick JM, Khodabakhsh AJ, Josephberg RG. Acute postoperative endophthalmitis caused by Actinomyces neuii. Am J Ophtalmol. 2002;133(1):145-7.

23. Acevedo F, Baudrand R, Letelier LM, Gaete P. Actinomycosis: a great pretender. Case reports of unusual presentations and a review of the literature. Int J Infect Dis. 2008;12(4):358-62.

24. van Mook W, Simonis F, Schneeberger P, van Opstal J. A rare case of disseminated actinomycosis caused by Actinomyces meyeri. Neth J Med. 1997;51(1):39-45.

25. Wong VK, Turmezei TD, Weston VC. Actinomycosis. BMJ. 2011;343:d6099.

26. Walther K, Bruder E, Goldenberger D, Mayr J, Schaad UB, Ritz N. Actinomyces neuii isolated from a 20-month-old girl with cervical lymphadenitis. J Pediatric Infect Dis Soc. 2015;4(3):e-32-7.

27. Mann C, Dertinger S, Hartmann G, Schurz R, Simma B. Actinomyces neuii and neonatal sepsis. Infection. 2002;30(3):178-80.

28. Knee DS, Christ MJ, Gries DM, Thompson MW. Actinomyces species and cerclage placement in neonatal sepsis: a case report. J Perinatol. 2004;24(6):389-91.

29. Chaudhry SI, Greenspan JS. Actinomycosis in HIV infection: a review of a rare complication. Int J STD AIDS. 2000;11(6):349-55.

30. Chen CY, Chen YC, Tang JL, Lin WC, Su IJ, Tien HF. Splenic actinomycotic abscess in a patient with acute myeloid leukemia. Ann Hematol. 2002;81(9):532-4. 
31. Dominguez DC, Antony SJ. Actinomyces and Nocardia infections in immunocompromised and nonimmunocompromised patients. J Natl Med Assoc. 1999;91(1):35-9.

32. Fry RD, Birnbaum EH, Lacey DL. Actinomyces as a cause of recurrent perianal fistula in the immunocompromised patient. Surgery. 1992;111(5):591-4.

33. Merle-Melet M, Mory F, Stempfel B, Maurer P, Regent D, Parent S, et al. Actinomyces naeslundii, acute cholecystitis, and carcinoma of the gallbladder. Am J Gastroenterol. 1995;90(9):1530-1.
34. Valour F, Sénéchal A, Dupieux C, Karsenty J, Lustig S, Breton P, et al. Actinomycosis: etiology, clinical features, diagnosis, treatment, and management. Infect Drug Resist. 2014;7:183-97.

35. Smith AJ, Hall V, Thakker B, Gemmell CG. Antimicrobial susceptibility testing of Actinomyces species with 12 antimicrobial agents. J Antimicrob Chemother. 2005;56(2):407-9.

36. Lerner PI. Susceptibility of pathogenic actinomycetes to antimicrobial compounds. Antimicrob Agents Chemother. 1974;5(3):302-9. 